\title{
ANALYTICITY OF THE SUSCEPTIBILITY FUNCTION FOR UNIMODAL MARKOVIAN MAPS OF THE INTERVAL.
}

by Yunping Jiang* and David Ruelle**.

$$
\begin{aligned}
& \text { Abstract. We study the expression (susceptibility) } \\
& \qquad \Psi(\lambda)=\sum_{n=0}^{\infty} \lambda^{n} \int_{I} \rho(d x) X(x) \frac{d}{d x} A\left(f^{n} x\right)
\end{aligned}
$$

where $f$ is a unimodal Markovian map of the interval $I$, and $\rho=$ $\rho_{f}$ is the corresponding absolutely continuous invariant measure. We show that $\Psi(\lambda)$ is analytic near $\lambda=1$, where $\Psi(1)$ is formally the derivative of $\int_{I} \rho(d x) A(x)$ with respect to $f$ in the direction of the vector field $X$.

\footnotetext{
* Math. Dept., Queens College of CUNY, Flushing, NY 11367 and Math. Dept., Graduate Center of CUNY and Math. Inst., AMSS, CAS. email: yunqc@forbin.qc.edu ** Math. Dept., Rutgers University, and IHES. 91440 Bures sur Yvette, France. email: ruelle@ihes.fr
} 
In a previous note $[\mathrm{Ru}]$ the susceptibility function was analyzed for some examples of maps of the interval. The purpose of the present note is to give a concise treatment of the general unimodal Markovian case (assuming $f$ real analytic). We hope that it will similarly be possible to analyze maps satisfying the Collet-Eckmann condition. Eventually, as explained in $[\mathrm{Ru}]$, application of a theorem of Whitney [Wh] should prove differentiability of the map $f \mapsto \rho_{f}$ restricted to a suitable set.

\section{Setup}

Let $I$ be a compact interval of $\mathbf{R}$ and $f: I \rightarrow I$ be real analytic. We assume that there is $c$ in the interior of $I$ such that $f^{\prime}(0)=0, f^{\prime}(x)>0$ for $x<c, f^{\prime}(x)<0$ for $x>c$, and $f^{\prime \prime}(c)<0$. Replacing $I$ by a possibly smaller interval, we assume that $I=[a, b]$ where $a=f^{2}(c), b=f(c)$. We assume that the postcritical orbit $P=\left\{f^{n} c: n \geq 1\right\}$ is finite: $P=\left\{p_{1}, \ldots, p_{m}\right\}$; in particular, $f$ is Markovian. We shall assume that $f$ is analytically expanding in the sense of Assumption A below; in particular the periodic orbits of $f$ are assumed to be repelling, and therefore $c$ cannot be periodic. We also assume that $f$ is topologically mixing [this can always be achieved by replacing $I$ by a smaller interval and $f$ by some iterate $f^{N}$ ].

\section{Theorem.}

Under the above conditions, and Assumption $A$ stated later, there is a unique $f$ invariant probability measure $\rho$ absolutely continuous with respect to Lebesgue on $I$. If $X$ is real analytic on $I$, and $A \in \mathbf{C}^{1}(I)$, then

$$
\Psi(\lambda)=\sum_{n=0}^{\infty} \lambda^{n} \int_{I} \rho(d x) X(x) \frac{d}{d x} A\left(f^{n} x\right)
$$

extends to a meromorphic function in $\mathbf{C}$, without pole on $\{\lambda:|\lambda|=1\}$.

\section{Change of variable}

The finite set $\{c\} \cup P$ decomposes $I$ into $m$ subintervals $I_{j}$, with $2 m$ endpoints (we "double" the endpoints of consecutive subintervals, distinguishing between a - endpoint at the right of an interval, and a + endpoint at the left). Note that $\eta=\left\{I_{j}: j=1, \ldots, m\right\}$ is a Markov partition for the map $f$. Consider the critical values of $f^{n}$. Then for large $n>0$, the set of critical values will be stabilized and is always $P$. We define polar endpoints as follows:

(1) $p \in P$ is a polar -endpoint of an interval in $\eta$ if $p$ is local maximum value of $f^{n}$ for $n$ large.

(2) $v \in P$ is a polar +endpoint of an interval in $\eta$ if $p$ is local minimum value of $f^{n}$ for $n$ large.

Every $p \in P$ is a polar - or +endpoint and may be both, $c$ is a nonpolar endpoint on both sides. 
We define now an increasing continuous map $\varpi: I \rightarrow \mathbf{R}$ so that $J=\varpi I$ is a compact interval. We write $\varpi I_{j}=J_{j}$ for $1 \leq j \leq m$. Denote by $\omega$ the inverse of $\varpi$. We assume that $\omega \mid J_{j}$ extends to a holomorphic function in a complex neighborhood of $J_{j}$ for $1 \leq j \leq m$ and that for $q \in\{c\} \cup P, \omega$ has the property

$$
\omega(\varpi q \pm \xi)=\omega(\varpi q) \pm \frac{\xi^{2}}{2}+O\left(\xi^{4}\right)
$$

if $q$ is a \pm polar endpoint, and

$$
\omega(\varpi q \pm \xi)=\omega(\varpi q) \pm \xi+O\left(\xi^{2}\right)
$$

if $q$ is a nonpolar endpoint. [We should really consider disjoint copies of the $I_{j}$ and $J_{j}$, and disjoint neighborhoods of these in $\mathbf{C}$ or in a Riemann surface two-sheeted near polar endpoints. This would lead to notational complications that we prefer to omit].

Applications of this singular change of coordinate have been used in [Ji1], [BJR], and $[\mathrm{Ru}]$; the reference [Ji2] contains some more relevant study regarding the method of singular change of coordinates in one-dimensional dynamical systems. The reader is encouraged to compare this method with orbifold metrics in [Th, Chapter 13]. Another relevant application of this method in complex dynamical systems can be found in $[\mathrm{DH}]$.

From now on we shall say that $\varpi q$ is a \pm polar (nonpolar) endpoint if $q$ is \pm polar (nonpolar).

\section{The dynamical system viewed after the change of variable.}

For any two intervals $I_{j}, I_{k} \in \eta$ with $f I_{j} \supset I_{k}$, we define

$$
\psi_{j k}=\varpi \circ\left(f \mid I_{j}\right)^{-1} \circ\left(\omega \mid J_{k}\right)
$$

Note that the $\psi_{j k}$ are restrictions of inverse branches of $g=\varpi \circ f \circ \omega: J \rightarrow J$ to intervals in $\eta$. The function $\psi_{j k}: J_{k} \rightarrow J_{j}$ extends holomorphically to a complex neighborhood of $J_{k}$. Indeed, note that $\left(f \mid I_{j}\right)^{-1}$ is holomorphic except if $I_{j}$ is one of the two intervals around $c$, in which case the singularity is corrected by $\omega \mid I_{n}$, where $I_{n}$ is the rightmost interval in $\eta$. In other cases $\omega \mid I_{k}$ cancels the singularity of $\varpi \mid I_{j}$ by our definition of $\omega$. [Note that $\psi_{j k}^{\prime}(x) \geq 0$ or $\leq 0$ on $J_{k}$ and may vanish only at an interval endpoint].

\section{Assumption A.}

Each $J_{k}$, for $k=1, \ldots, m$, has a bounded open connected neighborhood $U_{k}$ in $\mathbf{C}$ such that $\psi_{j k}: J_{k} \rightarrow J_{j}$ extends to a continuous function $\psi_{j k}: \bar{U}_{k} \rightarrow \mathbf{C}$ holomorphic in $U_{k}$, and with $\psi_{j k} \bar{U}_{k} \subset U_{j}$.

One checks that the sets $U_{k}$ can be assumed to be in $\epsilon$-neighborhoods of the $J_{k}$. Also, Assumption A implies that periodic points for $g$ are strictly repelling. The smoothness of $\omega, \varpi$ in the interior of subintervals shows that the same property holds for $f$, apart from interval endpoints where we however also assume the property to hold:

The periodic orbits of $f$ are strictly repelling. 


\section{Markovian graph.}

Consider the Markov partition $\eta=\left\{I_{j}\right\}$. Let us write $j \succ k\left(j\right.$ covers $k$ ) if $f I_{j} \supset I_{k}$ (we allow $j \succ j$ ). This defines a directed graph with vertex set $\{1, \ldots, m\}$ and oriented edges $(j, k)$ for $j \succ k$. Since we have assumed our dynamical system $f$ to be topological mixing, our graph is also mixing in the sense that there is $N \geq 1$ such that for all $j, k \in\{1, \ldots, m\}$ we have $j \succ \ldots \succ k$ (N edges) corresponding to $f^{N} I_{j} \supset I_{k}$.

\section{Transfer operators.}

For a function $\Phi=\left(\Phi_{j}\right)$ defined on $\sqcup J_{j}$, write

$$
\begin{gathered}
(\mathcal{L} \Phi)_{k}(z)=\sum_{j: j \succ k} \operatorname{sgn}(j) \psi_{j k}^{\prime}(z) \Phi\left(\psi_{j k} z\right) \\
\left(\mathcal{L}_{0} \Phi\right)_{k}(z)=\sum_{j: j \succ k} \operatorname{sgn}(j) \Phi\left(\psi_{j k} z\right)
\end{gathered}
$$

where $\operatorname{sgn}(j)$ is +1 if $\psi_{j k}$ is increasing on $J_{k}$, and -1 if $\psi_{j k}$ is decreasing on $J_{k}$. If $H$ is the Hilbert space of functions on $\sqcup_{j \in L} \bar{U}_{j}$ which are square integrable with respect to Lebesgue, and have holomorphic restrictions to the $U_{j}$, then $\mathcal{L}$ and $\mathcal{L}_{0}$ acting on $H$ are holomorphy improving, hence compact and of trace class.

\section{Properties of $\mathcal{L}$.}

For $x \in J_{k}$ we have

$$
(\mathcal{L} \Phi)_{k}(x)=\sum_{j \succ k}\left|\psi_{j k}^{\prime}(x)\right| \Phi_{j}\left(\psi_{j k} x\right)
$$

hence $\Phi \geq 0$ implies $\mathcal{L} \Phi \geq 0(\mathcal{L}$ preserves positivity) and

$$
\int_{J} d x(\mathcal{L} \Phi)(x)=\sum_{k} \int_{J_{k}} d x(\mathcal{L} \Phi)_{k}(x)=\sum_{j} \int_{J_{j}} d x \Phi_{j}(x)=\int_{J} d x \Phi(x)
$$

$\left(\mathcal{L}\right.$ preserves mass). Using mixing one finds that $\mathcal{L}$ has a simple eigenvalue $\mu_{0}=1$ corresponding to an eigenfunction $\sigma_{0}>0$. The other eigenvalues $\mu_{\ell}$ satisfy $\left|\mu_{\ell}\right|<1$, and their (generalized) eigenfunctions $\sigma_{\ell}$ satisfy $\int_{J} d x \sigma_{\ell}(x)=0$. If we normalize $\sigma_{0}$ by $\int_{J} d x \sigma_{0}(x)=1$, then $\sigma_{0}(d x)=\sigma_{0}(x) d x$ is the unique $g$-invariant probability measure absolutely continuous with respect to Lebesgue on $J$. In particular, $\sigma_{0}(x) d x$ is ergodic.

Let now $H_{1} \subset H$ consist of those $\Phi=\left(\Phi_{k}\right)$ such that the derivative $\Phi^{\prime}$ vanishes at the (polar) endpoints $\varpi a, \varpi b$ of $J$, and such that at the common endpoint $\varpi q(q \in$ $\{c\} \cup P \backslash\{a, b\}))$ of two subintervals we have equality on both sides of a quantity which is either

- the value of $\Phi$ for a nonpolar endpoint, or

- the value of $\pm \Phi^{\prime}$ for a polar \pm endpoint. 
We note that $\mathcal{L} H_{1} \subset H_{1}$ [this requires a case by case discussion]. Furthermore $\sigma_{0} \in H_{1}$ [take $\phi \in H$ such that $\phi \geq 0, \int_{J} d y \phi(y)=1$, and $\phi, \phi^{\prime}$ vanish at subinterval endpoints; then $\phi \in H_{1}$ and $\left.\sigma_{0}=\lim _{n \rightarrow \infty} \mathcal{L}^{n} \phi \in H_{1}\right]$.

Evaluating $\Psi(\lambda)$.

The image $\rho(d x)=\rho(x) d x$ of $\sigma_{0}(y) d y$ by $\omega$ is the unique $f$-invariant probability measure absolutely continuous with respect to Lebesgue on $I$. We have

$$
\rho(x)=\sigma_{0}(\varpi x) \varpi^{\prime}(x)
$$

Consider now the expression

$$
\Psi(\lambda)=\sum_{n=0}^{\infty} \lambda^{n} \int_{I} \rho(d x) X(x) \frac{d}{d x} A\left(f^{n} x\right)
$$

where we assume that $X$ extends to a holomorphic function in a neighborhood of each $I_{k}$ and takes the same value at both sides of common endpoints of intervals in $\eta$ (continuity). Also assume that $A \in \mathcal{C}^{1}(I)$. For sufficiently small $|\lambda|$, the series defining $\Psi(\lambda)$ converges. Writing $B=A \circ \omega$ ( $B$ has piecewise continuous derivative) and $x=\omega y$ we have

$$
X(x) \frac{d}{d x} A\left(f^{n} x\right)=X(\omega y) \frac{1}{\omega^{\prime}(y)} \frac{d}{d y} B\left(g^{n} y\right)
$$

hence

$$
\Psi(\lambda)=\sum_{n=0}^{\infty} \lambda^{n} \int_{J} d y \sigma_{0}(y) \frac{X(\omega y)}{\omega^{\prime}(y)} \frac{d}{d y} B\left(g^{n} y\right)
$$

Defining $Y(y)=\sigma_{0}(y) X(\omega y) / \omega^{\prime}(y)$, we see that $Y$ extends to a holomorphic function in a neighborhood of each $J_{k}$, which we may take to be $U_{k}$, except for a simple pole at each polar endpoint of $J_{k}$. Since $\sigma_{0} \in H_{1}$, the properties assumed for $\omega$ imply that also $(X \circ \omega) \times \sigma_{0} \in H_{1}$. Note that near a nonpolar subinterval endpoint $\varpi q$

$$
\omega^{\prime}(\varpi q \pm \xi)=1+O(\xi)
$$

and near a \pm polar endpoint

$$
\omega^{\prime}(\varpi q \pm \xi)=\xi+O\left(\xi^{3}\right)
$$

Therefore

$$
Y(\varpi q \pm \xi)=A^{ \pm} \frac{1}{\xi}+B^{ \pm}+O(\xi)
$$

where $B^{+}=B^{-}$for the two sides of $\varpi q$, and $B^{+}=0$ at the left endpoint $\varpi a$ of $J, B^{-}=0$ at the right endpoint $\varpi b$ of $J$. We may write

$$
\int_{J} d y \sigma_{0}(y) \frac{X(\omega y)}{\omega^{\prime}(y)} \frac{d}{d y} B\left(g^{n} y\right)=\int_{J} d y Y(y) g^{\prime}(y) \cdots g^{\prime}\left(g^{n-1} y\right) B^{\prime}\left(g^{n} y\right)
$$




$$
=\int_{J} d s\left(\mathcal{L}_{0}^{n} Y\right)(s) B^{\prime}(s)
$$

where $\mathcal{L}_{0}$ has been defined above, and we have thus

$$
\Psi(\lambda)=\sum_{n=0}^{\infty} \lambda^{n} \int_{J} d s\left(\mathcal{L}_{0}^{n} Y\right)(s) B^{\prime}(s)
$$

\section{Properties of $\mathcal{L}_{0}$.}

We let now $H_{0} \subset H$ be the space of functions $\Phi=\left(\Phi_{k}\right)$ vanishing at the endpoints $\varpi a, \varpi b$ of $J$, and such that the values of $\Phi$ on both sides of common endpoints of intervals $J_{j}$ coincide (continuity). Therefore $\mathcal{L}_{0} H_{0} \subset H_{0}$.

There is a periodic orbit $\gamma_{1}, \ldots, \gamma_{p}\left(\right.$ with $\left.g \gamma_{j}=\gamma_{j+1(\bmod p)}\right)$ of polar endpoints where $\gamma_{\alpha}$ is the \pm endpoint of some subinterval $J_{k(\alpha)}$. Choose $P_{\alpha}$ to be 0 on subintervals different from $J_{k(\alpha)}$, and to be holomorphic on a complex neighborhood of $J_{k(\alpha)}$ except at $\gamma_{\alpha}$. Also assume that

$$
P_{\alpha}\left(\gamma_{\alpha} \pm \xi\right)=\frac{1}{\xi}+O(\xi)
$$

and that $P_{\alpha}$ vanishes at the endpoint of $J_{k(\alpha)}$ different from $\gamma_{\alpha}$. Then

$$
\mathcal{L}_{0} P_{\alpha}-\mid f^{\prime}\left(\left.\gamma(\alpha)\right|^{1 / 2} P_{\alpha+1(\bmod p)} \in H_{0}\right.
$$

Therefore $\mathcal{L}_{0}^{p} P_{1}-\Lambda P_{1}=u \in H_{0}$ where $\Lambda=\prod_{\alpha=1}^{p} \mid f^{\prime}\left(\left.\gamma(\alpha)\right|^{1 / 2}>1\right.$. Since the spectrum of $\mathcal{L}$ acting on $H$ is contained in the closed unit disk, and since the derivative $u^{\prime}$ is in $H$, we may define $v=\left(\mathcal{L}^{p}-\Lambda\right)^{-1} u^{\prime} \in H$. Since $\int_{J} d y u^{\prime}(y)=0$ we also have $\int_{J} d y v(y)=0$ and we can take $w \in H_{0}$ such that $w^{\prime}=v$. We have thus

$$
\left(\left(\mathcal{L}_{0}^{p}-\Lambda\right) w\right)^{\prime}=\left(\mathcal{L}^{p}-\Lambda\right) w^{\prime}=\left(\mathcal{L}^{p}-\Lambda\right) v=u^{\prime}
$$

so that $\left(\mathcal{L}_{0}^{p}-\Lambda\right) w=u$ there is no additive constant of integration since $\left(\mathcal{L}_{0}^{p}-\Lambda\right) w$ and $u$ are in $H_{0}$ ]. Finally

$$
\left(\mathcal{L}_{0}^{p}-\Lambda\right)\left(P_{1}-w\right)=0
$$

There is thus a $\mathcal{L}_{0}$-invariant $p$-dimensional vector space spanned by vectors $P_{\alpha}-w_{\alpha}$ with $w_{\alpha} \in H_{0}$, such that the spectrum of $\mathcal{L}_{0}$ restricted to this space consists of eigenvalues $\omega_{\ell}$ with

$$
\omega_{\ell}=\Lambda^{1 / p} e^{2 \pi \ell i / p}=\left|\prod_{\alpha=1}^{p} f^{\prime}\left(\gamma_{\alpha}\right)\right|^{1 / 2 p} e^{2 \pi \ell i / p}
$$

for $\ell=0, \ldots, p-1$.

For the postcritical but nonperiodic polar points $\tilde{\gamma}_{1}, \ldots, \tilde{\gamma}_{q}$ define $\tilde{P}_{\beta}$ like $P_{\alpha}$ above, with $\gamma_{\alpha}$ replaced by $\tilde{\gamma}_{\beta}$. For each $\beta$ there is $\alpha=\alpha(\beta)$ with

$$
\mathcal{L}_{0}^{q}\left(\tilde{P}_{\beta}-\tilde{\Lambda}_{\beta} P_{\alpha}\right) \in H_{0}
$$


with some $\tilde{\Lambda}_{\beta} \neq 0$, hence

$$
\mathcal{L}_{0}^{q}\left(\tilde{P}_{\beta}-\tilde{\Lambda}_{\beta}\left(P_{\alpha}-w_{\alpha}\right)\right)=\tilde{Y}_{\beta} \in H_{0}
$$

\section{Poles of $\Psi(\lambda)$.}

We may now write

$$
Y=Y_{0}+Y_{1}+Y_{2}
$$

where

$$
\begin{gathered}
Y_{0} \in H_{0} \\
Y_{1}=\sum_{\alpha=1}^{p} c_{\alpha}\left(P_{\alpha}-w_{\alpha}\right) \\
Y_{2}=\sum_{\beta=1}^{q} \tilde{c}_{\beta}\left(\tilde{P}_{\beta}-\tilde{\Lambda}_{\beta}\left(P_{\alpha(\beta)}-w_{\alpha(\beta)}\right)\right)
\end{gathered}
$$

and there is a corresponding decomposition $\Psi(\lambda)=\Psi_{0}(\lambda)+\Psi_{1}(\lambda)+\Psi_{2}(\lambda)$. Here $\Psi_{1}(\lambda)$ is a sum of terms $C_{\ell} /\left(\lambda-\omega_{\ell}\right)$ where $\omega_{\ell}=\Lambda^{1 / p} \times p$-th root of unity; $\Psi_{2}(\lambda)=$ polynomial of degree $q-1$ in $\lambda$ plus $\lambda^{q} \sum_{\beta=1}^{q} \tilde{c}_{\beta} \tilde{\Psi}_{\beta}(\lambda)$ where $\tilde{\Psi}_{\beta}$ is obtained if we replace $Y$ by $\tilde{Y}_{\beta}$ in the definition of $\Psi$. The poles of $\Psi(\lambda)$ are thus those of $\Psi_{1}(\lambda)$ at the $\omega_{\ell}$ and those of $\Psi_{0}(\lambda)$ and $\tilde{\Psi}_{\beta}(\lambda)$. The discussion is the same for $\Psi_{0}$ and the $\tilde{\Psi}_{\beta}$, we shall thus only consider $\Psi_{0}$. Since $Y_{0} \in H_{0}$ and $\mathcal{L}_{0} H_{0} \subset H_{0}$ we have

$$
\begin{gathered}
\Psi_{0}(\lambda)=\sum_{n=0}^{\infty} \lambda^{n} \int_{J} d s\left(\mathcal{L}_{0}^{n} Y_{0}\right)(s) B^{\prime}(s)=-\sum_{n=0}^{\infty} \lambda^{n} \int_{J} d s\left(\mathcal{L}_{0}^{n} Y_{0}\right)^{\prime}(s) B(s) \\
=-\sum_{n=0}^{\infty} \lambda^{n} \int_{J} d s\left(\mathcal{L}^{n} Y_{0}^{\prime}\right)(s) B(s)
\end{gathered}
$$

It follows that $\Psi_{0}(\lambda)$ extends meromorphically to $\mathbf{C}$ with poles at the $\mu_{k}^{-1}$. We want to show that the residue of the pole at $\mu_{0}^{-1}=1$ vanishes. Since $\int_{J} d y \sigma_{k}(y)=0$ for $k \geq 1$, the coefficient of $\sigma_{0}$ in the expansion of $Y_{0}^{\prime}$ is proportional to

$$
\int_{J} d y Y_{0}^{\prime}(y)=Y(\varpi b)-Y(\varpi a)=0
$$

because $Y_{0} \in H_{0}$. Therefore $\Psi_{0}(\lambda)$ is holomorphic for $|\lambda|=1$, and the same holds for the $\tilde{\Psi}_{\beta}(\lambda)$, concluding the proof of the theorem. In fact we know that the poles of $\Psi(\lambda)$ are located at $\mu_{k}^{-1}$ for $k \geq 1$, and at $\omega_{\ell}^{-1}$ for $\ell=0, \ldots, p-1$, so that $\left|\mu_{k}^{-1}\right|>1,\left|\omega_{\ell}^{-1}\right|<1$.

\section{Acknowledgments.}

One of us (Y.J.) is partially supported by grants from the NSF and the PSC-CUNY Program, and by the Hundred Talents Program of the CAS. 


\section{References.}

[BJR] V. Baladi, Y. Jiang, H.H. Rugh. "Dynamical determinants via dynamical conjugacies for postcritically finite polynomials." J. Statist. Phys. 108, 973-993(2002).

[DH] D. Douady and J. H. Hubbard, "A proof of Thurston's topological characterization of rational functions." Acta Math., Vol. 171, 263-297(1993).

[Ji1] Y. Jiang, "On Ulam-von Neumann transformations." Commun. Math. Phys., 172, 449-459(1995).

[Ji2] Y. Jiang, "Renormalization and Geometry in One-Dimensional and Complex Dynamics." Advanced Series in Nonlinear Dynamics, Vol. 10 (1996), World Scientific Publishing Co. Pte. Ltd., River Edge, NJ.

$[\mathrm{Ru}]$ D. Ruelle. "Differentiating the absolutely continuous invariant measure of an interval map $f$ with respect to $f$." Commun. Math. Phys. to appear.

[Th] W. Thurston, "The Geometry and Topology of Three-Manifolds", Electronic version 1.1-March 2002, http://www.msri.org/publications/books/gt3m/

[Wh] H. Whitney. "Analytic expansions of differentiable functions defined in closed sets." Trans. Amer. Math. Soc. 36,63-89(1934). 